\title{
智能化家居对于室内设计的适应性发展探究
}

邱斌

深圳市浩天装饰有限公司

DOI:10.32629/btr.v2i8.2393

[摘 要] 现代社会科技快速发展, 人们的生活水平逐渐提高,对室内设计的要求也更加高,这就要求目前的室内设计必须能够 满足消费者对室内设计的要求, 只有这样, 室内设计才能更好的发展。这就迫使室内设计创造出一种新颖的技术以满足需要, 智能家居系统应运而生,其作为一种新颖的室内设计技术,优点非常明确,在室内设计中的地位越来越重要。本文先介绍智能化 家居的发展以及特点,进而分析其对于室内设计的适应性发展,得出结论,提出室内设计中如何正确使用智能化家居为人们服 务,从而向人们推广使用智能化家居。

[关键词] 智能化; 家居设计; 室内设计; 适应性

现阶段, 人们普遍追求更舒适、更便捷的室内生活, 因此 对家居的设计也提出了更高的要求, 智能家居系统是一种新 型的科技技术, 将其应用到室内设计中, 能极大提升空间的 舒适性与便捷性, 但是因为技术以及其他因素的限制, 智能 家居在室内设计中的应用还欠缺协调。因此, 将智能化家居 系统与美学艺术、设计技术有机结合, 使其适应现代室内设 计的特征, 是室内设计目前急需解决的问题。

\section{1 智能家居与室内设计}

科技的有效发展为人们的生活带来了极大的便捷, 为人 们创造了丰富多姿的生活, 智能家居的出现, 为室内设计的 发展新增了一股活力, 而其诞生也是预示着今后科技住宅一 个重要的发展目标, 因此智能家居这一理念的产生不但能够 完全地呈现出一种强烈的时代性, 并且也是一种优质的新型 设计理念。这两个因素的实现, 不但是要以综合性的思维进 行思考其在物质方面的需求, 并且也要以认真、重视的态度 进行调整艺术、技术二者之间的协调性以及融合性, 所以, 在对其设计的过程中, 一定要充分地发挥材料具有的功能、 施工工艺的特点以及先进的物联网科技所具有的功能。以宏 观的思维进行分析, 如今的室内设计已经慢慢地创建了装潢 设计一环境设计一生活设计三个主要步骤紧密且依次连接 式的发展模式。从整体上看, 现阶段的智能家居设计包含有 以下几点特征:

\section{1 关注使用者的身心体验}

人作为室内空间的使用主体, 对于评判智能住宅是非常 具有话语权的。作为智能家居其营造的就是简便、舒适、健 康、绿色环保的居住环境, 这样一来能够极大地提高人们的 身心体验, 更加能够促进人们的居住质量。具体应当从以下 三点进行关注:

1.1.1智能住宅应当具备合理控制周围环境的能力, 例 如: 湿度、温度等。

1.1.2智能住宅不应当仅仅只注重智能化, 也应当同室 内设计风格相吻合。

1. 1.3 智能住宅应当符合居住者日常居住使用习惯, 只
有这样才能从最大程度上满足人们对智能住宅的居住要求。

\section{2 艺术同科技相结合}

室内设计不仅关乎人们的生活更应当能够体现出居住者 的审美情趣, 应当是技术结合艺术的产物, 这背后应当是结合 了许多当代的新型材料及施工技术, 实现人们的设想及需求。 室内设计作为一个集合视听娱乐、居住、学习、餐饮等多种 设计的场所, 不再是简单的空间装饰, 更应当是一个蕴含各种 复杂系统的营造过程, 使得室内最终成为一个能够体现综合 的空间艺术, 装饰陈设艺术及现代科技的最为理想场所。

1.3 应当具备一定的前瞻性

伴随着科技的发展及人们生活水平的提高, 室内设计更 新周期趋短, 很多新兴事物快速融入到人们的生活中去, 人 们对于室内设计风格的变化以及居住的功能变化也变得更 加活跃, 所以, 关于智能家居设计的思维应当更具有前瞻性 更加跳跃, 并且可以将可持续设计理念融入到日常设计当中 去, 这样设计才能够顺应当前的发展形势。

1. 4提升住宅的品质及附加值

智能家居所创造的家居居住环境可以说是应用了大量 的新材料、监测系统、自动化控制技术以及网络技术等, 尤 其是一些材料五金件等都具备相当高的科技含金量, 施工技 术当然也不拘泥于当前的传统工艺, 这些给室内设计带来的 附加值都是我们有目共睹的。

1.5 体现居住者的个性需求

相信每一个住宅的主人都有其不同的个性, 个性能够表 明一个人的价值观、世界观以及思维方式, 能够通过一定的 再提体现人们的审美。室内空间是人们日常生活较为主要的 场所, 必须应当充分尊崇使用者的个人需要, 避免同质化的 情况发生, 这样才能够全面细致的反映出主人的审美情趣及 态度。

\section{2 智能家居设计思维}

2. 1 实现住宅设计生态化

在我国社会经济发展中, 实现制造方便的同时, 也在很 大程度上损害自然环境, 在很大程度上浪费各项能源, 基于 
此, 人们在进行室内设计过程中必须进一步重视绿色环保理 念, 同时各行业也对其实现了进一步重视。所以相关行业工 作人员必须重视生态住宅和绿色住宅建设, 从而在很大程度 上实现节能环保, 从而目前生态化建设也成为住宅建设的必 然发展趋势。在进行具体设计过程中必须确保, 进一步满足 业主对生活及居住环境的各项需求, 同时在进行设计作业时 有效结合审美与舒适度。与此同时, 相关工作者还需要科学 处理水资源应用和各项能源应用, 同时实现能源利用率的有 效提升, 进一步推进绿色住宅建设。

\section{2 实现设计以人为本}

从本质而言, 智能家居服务具体工作对象为人, 因此在 设置系统服务作业时必须实现以人为本, 相关内容的设置也 必须与人类生活完全符合。也就是说, 家居服务系统这很大 程度上受到业主生活习惯的直接影响。与此同时, 家具设计 工作在很大程度上也必须基于不同群体具体需求进行作业, 必须充分考虑到残疾人贞幼儿等特殊群体, 确保实现家具设 计以人为本, 在很大程度上推进我国智能家居建设, 从而家 居系统设计更加符合人体需求。

\section{3实现设计个性化}

在进行居住环境具体设计时, 通常会受到多种环境干扰, 包括业主年龄层次, 行为习惯, 生活理念以及消费能力等, 以 上因素的不同会在一定程度上影响业主居住需求。基于此, 在进行具体室内设计工作中, 需要针对不同人群进行具体作 业。而在进行智能模块组合作业时必须基于应用人群具体需 求进行灵活组建。智能化控制软件在不同场所也具有较高差 异性, 相关工作人员可以基于具体需求进行自由组网, 通过 分析使用人群具体需求选择完善方案和初级方案, 确保居住 环境有更高的市场价值和发展前景。

2. 4 实现产品多样化

在进行智能家居设计作业时, 主要基于智能终端实现其 应用高效性与便捷性。包括室内新风系统, 气候调节系统以 及各种智能识别系统。与此同时, 产品不仅需要实现功能多 样性, 同时其造型也需要满足不同风格需求, 确保相关产品 能够有效融入装饰风格, 例如在进行墙纸设计时, 需要基于 室内环境的变化实现墙纸颜色变化, 不仅能够使室内相关设 备能够有效感知室内具体环境, 同时还能够使其更好地符合 人们心理需求。在进行具体产品设计时, 一定要摒弃传统的 单调乏味, 尽量使其有效融入室内陈设, 使建筑装饰造型更 好的匹配室内环境。

\section{3 智能家居的应用思维}

3.1 体验全方位人性化
智能家居先进的优点不能只体现在宣传上, 应当注重客 户的使用体验, 实现一键式控制, 操作简单易懂。比如, 按动 遥控就能一次关闭所有电器和电灯, 快捷省事节能。实现这 一功能最理想的操作方法就是, 按 “场景” 模式, 包括离家、 回家、影院、睡觉等分类。

3. 2 智能家居标准的统一

企业为了增加自己产品的 “含金量” , 往往会制定各自 的智能家居网络接口标准和数据传输协议。尽管这样的协议 是支撑智能家居系统顺畅运行的关键, 然而不同企业的产品 不通用、不兼容, 无法融入到更高层次的控制系统中, 这样就 算市场规模不断扩大, 整个行业也难以做大做强。

3. 3模块集成化, 联动化, 操作简单化

智能化家居的硬件设备内部结构一定是科技尖端产品, 内部结构较为复杂, 然而体现在操作系统上的时候就应当做 到极简, 简化操作也是评价智能家居的一个标准。

3. 4 控制方式的多样化, 提高交互能力

住宅与人的交互能力是体现智能住宅 “智商” 的标志。 在智能化系统的控制下, 住宅是 “活” 的, 懂得交流, 学习, 记 忆, 改进。对于不具备智能控制属性的设备, 可以通过智能电 源等方式对设备进行智能化改造, 使其具备智能控制功能。

3. 5 成本合理化

住宅智能化系统的建造成本是室内设计中必须考虑的 重要因素。只有将住宅智能化系统的建设成本控制在一个比 较合理的, 可选择的范畴内, 才能为广大消费者接受, 产品接 了地气才会更有前进的动力。

\section{4 结束语}

科技住宅是未来建筑行业的一大发展趋势, 而科技住宅 的发展与实现意味着会有更多的智能家居、更完备的智能家 居系统被应用到建筑中, 因而智能家居对于室内设计的适应 性发展将是现代建筑设计急需解决的一个问题。结合现代人 的居住需求与审美要求, 笔者认为设计人员应当在注重居住 功能的同时突出造型设计, 在追求设计美感的同时做好绿色 设计, 以此实现室内设计与智能家居的协调发展。

\section{[参考文献]}

[1]陈康勇,陈昌泽.基于总线型的智能家居系统的设计 与实现 $[J]$.福建电脑,2017,33(11):27-28+43.

[2]郭晓敏,王耀民.新时代的住宅一探析智能化家居设计 [J].工业设计,2016,(03):97-98.

[3]张婷.推广应用家居智能化技术提高室内装修智能化 水平 $[J]$.智能建筑与智慧城市,2017,(01):44-45. 